\title{
Sinneswahrnehmungen nach indischen Bewertungen
}

\author{
Von Reinhold F. G. MüLleR
}

Obwohl viele wissenschaftliche Untersuchungen zu der gestellten Aufgabe durchgeführt worden sind, dürften doch noch manche unzureichend geklärten Ergebnisse bestehen, so daß eine gewisse, zusammenfassende Übersicht begrüdbar ist. Diese bemüht sich hier um eine medizingeschichtliche Darlegung, auch für den, welcher dem Bereich ferner steht oder sich nicht in die Texte selber einarbeiten kann. Zu letzten werden zwar Spracherklärungen tunlich eingeschränkt, lassen sich aber doch nicht ganz vermeiden. Ferner werden ärztliche Texte nicht bevorzugt, um eine Einsicht in allgemeine Bewertungen der Inder zu gewinnen.

Im Rgveda, der als älteste U̇berlieferung allgemein gilt, wird fast lehrmäßig erklärt in dem Lied an das «Jedermannsfeuer» (was etwa mit einem Seelenbegriff verglichen werden kann) I, 6, 5-6: ,Fest ist (dieses) Licht (im Herzen) eingesetzt, zumal zum Sehen; Denken (manas $\sim$ Geist $^{1}$ ) ist das schnellste unter allen, welche fliegen-lassen ${ }^{2}$. - Heraus lassen meine Ohrlöcher fliegen, heraus das Blikken, heraus dieses Licht, was im Herzen zugesetzt ist; heraus wandert mein Denken in die Ferne strahlend (und fragend), was werde ich wohl reden und an was werde ich denken ${ }^{3}{ }^{6}$ Dieser alte Textbeleg unterrichtet bereits sehr über die ursprüngliche Denkweise der vedischen Inder, gibt aber auch Anlaß zu verschiedenen Beurteilungen. Inhaltlich fällt auf, daß das Denken gleichsam den beiden erwähnten Sinneswahrnehmungen übergeordnet ist, und zwar ihren Äußerungen, welche ausdrücklich durch die Ausdrucksweise ,fliegen-lassen" wie zielmäßig geschildert erscheinen. Dabei entspricht die Bezeichnung Ohrlöcher einer unmittelbaren Beobachtung, hingegen das Blicken oder der Blick der ursprünglichen Ausdrucksform als ,Ausstrahler'. Entwicklungsgeschichtlich betrifft eine augenblickliche Anschauung die Wortbildung ,Ohrlöcher'. Das ,Blicken ${ }^{6}$ darf in der indischen Sprachwissenschaft bei einem Tätigkeitswort als Substantiv, ,was als Besonderheit gelten muß (visessya), aufgefaßt werden. Damit ist eine frühzeitige und durchlaufende Eigenart der alten Wissenschafter in Indien aufgezeigt, wenn von gelegentlichen anschaulichen Bedeutungen (wie bei den Ohrlöchern) abgesehen wird. Jedoch ein indischer Philologe widersprach dieser Wortbeurteilung: «aber die Grundbedeutung ist im Laufe der langen Sprachentwicklung vielfach vollkommen verblaßt»; dabei wird auch auf die «nicht ernst zu nehmenden Ety* mologien der indischen Exegeten» hingewiesen. Aber ein anderer Fachwissenschaftler fordert dazu eine Nachprüfung, denn sie «bildet eine dringende Aufgabe der indischen Philologie, die früher auf die, vorwissenschaftliche ${ }^{6}$ Methode

1 Nova Acta Leopoldina, N.F. Nr. 108 (Leipzig 1952).

${ }^{2}$ Die Sprache 7 (Wiesbaden-Wien 1961) 64.ff.

3 Mitt. Inst. Orientforsch. 6 (Berlin 1958) $266 \mathrm{ff}$. 
der einheimischen Grammatiker allzu selbstsicher herabgeblickt hat». Diesem Einwand ist zugestimmt worden, zumal bei dem Fachausdruck nirukti, der medizingeschichtlich eine ,Aussage ${ }^{6}$ [zur Wortbestimmung] bedeutet ${ }^{4}$.

Über den Sinn, dessen indische Bezeichnung als indriya «Kraft, Vermögen » ${ }^{5}$ bedeutet, finden sich vielfache Lehren in den Philosophemen im Rahmen der epischen Überlieferungen vom Mahäbhärata ${ }^{6}$, so auch abschließend in XII, 311, $16 \mathrm{ff}$. Hier wird gelehrt: ,Das Denken wandert - indem es durch die Sinnesvermögen gewandert wird (17). Aber nicht die Sinnesvermögen sehen, wirklich sieht das Denken nach (den Zielen). Das Blicken (cakșus) sieht die Ansichtsziele vermittels vom Denken, aber nicht vermittels vom Blicken (18). Wenn das Denken verwirrt ist, sieht das Blicken hin, aber sieht nicht. Ebenso sehen alle Sinnesvermögen, wie ausdrücklich erklärt wird. 'Auch hier sind die Sinnesvermögen dem Denken untergeordnet, und zwar mit deutlicher inhaltlicher Betonung. Ferner wird auffällig das Blicken hervorgehoben, wie als Muster der Sinnesvermögen oder diese beherrschend oder ersetzend. Die Bewertung kann als alt beurteilt werden. Denn schon im Rgveda, in dem Liede an den Wind, wird abschließend erklärt in X, 168, 4: ,sein Rauschen wird gehört, nicht sein Anblick ${ }^{6}$, weil ein fehlendes, wird gesehen" wegen der hohen Einschätzung vom Sehen selbstverständlich war.

Der Name Purāna entspricht zwar einer «alten Erzählung», aber die darnach überlieferten Texte haben mancherlei spätere Berichte aufgenommen, darunter auch solche aus ärztlichen Lehren ${ }^{7}$. Unter diesen ist Viṣnudharmottara-Puräna II, 115 zu bemerken, weil sein Text ein Vorbild hat im Agni-Puräna 370 ${ }^{8}$, zugleich ein Zeichen für eine breite Anteilnahme an derartigen Aufgaben, welche hier sich auf die Sinne beziehen [wobei das Ergänzungswort -vermögen der Einfachheit halber weiterhin fortgelassen wird]. Dazu führt Agni-Purāna $370 \mathrm{im} \mathrm{Be-}$ ginn ein:

(1) «The sensibilities of sound (hearing), touch, sight, taste, and smell, are the attributes of such sense organs, as the ears, the skin, the eyes, the tongue, and the nose.» So übersetzt der moderne Inder ${ }^{9}$ zu einem bequemen Verständnis, was jedoch nicht die eigentliche Bedeutung der Textworte wiedergibt. Es muß zwar eingeräumt werden, daß die Ausdrucksweise laienmäßig erscheint [was auch in ärztlichen Fachtexten nicht so selten beobachtet werden kann]; jedoch

4 Centaurus. 9 (Copenhagen 1963) 29-34.

5 Manfred Mayrhofer, Kurzgefaßtes etymologisches Wörterbuch des Altindischen, Band 1, S. 88-89 (Heidelberg 1956), ohne Erwähnung von indriya als Sinn.

6 Text-Edition by Ramechandrashasrti KinJawadekar (Poona 1929-1933).

7 M.Winternitz, Geschichte der indischen Literatur; vgl. die drei Indices (Leipzig 1909 bis 1922).

8 Anandāśrama Sanskrit Series 41 (Poona 1900).

9 M.N.Dutr, English Translation of Agni Puranam, S. 1291 (Calcutta 1903-1904). 
ihm muß nach Möglichkeit bei einer Übertragung nachgekommen werden: ,Das Mittel zum Horchen (śrotra $\sim \mathrm{Ohr}^{10}$ ), Haut (tvac), beide Blickende (cakșus), Zunge (jihvā), das Beriechende (ghräna) betreffen wohl die Sinne bezüglich der Wesen; Stimme, Getast, Anblick, Geschmack, Geruch sind wohl in Beginnen so die Eigenschaften. (2) After, Schoß, Hände, Füße, Sprache dürfte als Wirkung wohl ebenso entstehen; Entleerung, Wollust, Verhaltung, Gang, Sprache usw. ist so eine Wirkung. (3) Fünf Wirksinne dann und fünf Besinnungssinne und die Ziele der Sinne und die fünf Großwesen (mahäbhütäh), das Denken sind so die Herrscher. (4) Das Selbst, der Unentfaltete, die vierundzwanzig Wesenheiten (tattva ${ }^{11}$ ) ist der puruṣa (wörtlich «Mann» Seele) sowohl vereinigt als auch getrennt, wie die beiden Fische im Wasser.' Da springt der Text auf ein geistiges Gebiet über. Dabei ist zu berücksichtigen, daß in den Purāna-s regelmäßig ärztliche Grundsätze nur nebenher aufgenommen wurden, so auch sonst in AgniPurāṇa ${ }^{11}$. Immerhin erscheint dann beachtlich für eine Beurteilung, welche Anteile allgemein Aufmerksamkeit erweckten, welche eine gewisse Bevorzugung beanspruchen konnten. Für einen mehr erschöpfenden Einblick sind jedoch ärztliche Fachtexte zu nutzen.

Hierzu ist vor allem ein medizinisches Sammelwerk heranzuziehen, das sich besonders durch erkenntnismäßige Darlegungen auszeichnet, die Caraka-Sam$h i t \bar{a}^{12}$. In ihrem Lehr-Leitfaden, sūtra 8, 7, wird erklärt: „Die vom Denken ausströmenden Sinne werden zielend zum Ergreifen der Ziele. ${ }^{6}$ Hier ist grundsätzlich der Begriff von artha = Ziel dargelegt, wie er schon [zuvor] im Rgveda VI, 9, 5-6 angedeutet ist, bereits dort in Abhängigkeit vom Denken, und wie er dann weiterhin auch wissenschaftlich bewahrt wurde, gegensätzlich zu modernen Vorstellungen. Gewiß ist nicht zu bezweifeln, daß ursprünglich, auch gelegentlich weiterhin, ein Empfang von Sinnesreizen von der Umgebung her beachtet wurde ${ }^{13}$. Aber in der indischen grundsätzlichen Wertung setzte sich durch, daß das sogenannte Objekt doch subjektiv eingeschätzt wurde, was geschichtlich beachtlich ist. So wurde etwa auch im «Traumschlüssel des Jagaddeva » ${ }^{14}$, I, 3 der, wer «seine Sinne in der Gewalt hat» (yatendriyah), übertragen, wobei kritisch zu berücksichtigen ist, daß der Traum indisch als Wirklichkeit, sogar oft als krankheitsbewirkend ${ }^{15}$ galt.

10 Wackernagel-Devrunnwe, Altindische Grammatik, Band II, 2, §517a (Göttingen 1954).

11 Vgl. Archiv Orientální 31 (Praha 1963) S. 378 ff. oder Rocznik Orientallistyczny 28, in Druck (Warszawa 1964).

12 Text-Edition by Narendra NĀ̄tha ŚĀstrī (Lahore 1921).

13 Acta histor. scient. natural. medicin. 8 (Kopenhagen 1951) S. $27 \mathrm{ff}$.

14 J.v. Negeleiv (Gießen 1912).

15 Centaurus 2 (Copenhagen 1953) S. $189 \mathrm{ff}$. 
In Caraka-Samhitā sūtra ${ }^{\circ}$ 8, 7 ff. folgte dann eine aufschließende Liste von Benennungen der einzelnen Anteile der Sinnesbereiche: „Da sind Blicken (cakșus), Horchmittel (śrotra), Geruch (ghräna), Geschmack bewirkend (rasana), Betastung (sparśana) die fünf Sinne. 6

(8) ,Die fünf Sinnes-dravyas sind Raum (kha), Wind (vãyu), Licht-Feuer (jyotis), Gewässer (ap), Erde (bhūr). Die Bezeichnung dravya wird üblich als «Gegenstand, Substanz» oder dergleichen erklärt, bedeutet aber ihrer Ausdrucksbildung nach $d r u-=$ laufen den Fachbegriff, , was laufen muß ${ }^{6}{ }^{16}$, wie auch im Kommentar erläutert gemäß der Bedeutung der Entwicklung aus- und nach-einander der aufgezählten mahäbhütas ${ }^{17}$, welche die Welt- und Lebensanschauungen der Inder beherrschen. Es ist eigenartig, daß diese meist als Stufen einer Umwandlung gewertet wurden, aber auch als wirkende ,Groß-Wesen', so schon in Mahäbhärata XII, 310, 19 und ebenso [wie zuvor vermerkt] wie volkstümlich im Agni-Purāna 370, 3. Dabei ist auch zu berücksichtigen, daß der Begriff von dem oft sächlichen Ausdruck sattva $=$ Wesenheit als ein wirkender ,Wesentlicher ${ }^{6}$ bewahrt wurde, wie etwa in der Einleitung zu Vägbhatas Hrdyaya śärïra ${ }^{\circ} 1^{18}$.

(9) ,Die fünf Sinnesstandorte sind die beiden Augen ( $a k s ̦ i$ ), die beiden Ohrlöcher (karna), die beiden Nasenlöcher (nāsikā), die Zunge (jihvā), die Haut (tvac). ' In Standort = adhistāna ist durch adhi $=$ «über» nicht gesichert als Über-Stellung (sthāna) bezeichnet, weil adhi auch «in bezug auf $-»$ bedeutet. Jedenfalls ist eine Organform darnach nicht ausgedrückt. Gemäß der ausführlichen Augenheilkunde in Suśruta-Samhitā uttara ${ }^{\circ}$ 1-21 dürfen zwar unmittelbare Beobachtungen der Augenorgane angenommen werden, jedoch in der vermeintlichen «Anatomie» [welche nach Titel und Lehrinhalt als samkhyā eine Zusammenzählung oder Berechnung der körperlichen Anteile ist] werden die angeblichen Sinnesorgane in śārïra ${ }^{\circ}, 10$ als Strömungen (srotas) bezeichnet. ,Horchende (sravana $\sim \mathrm{Ohr})$, führende (nayana $\sim$ Auge), sprechende (vadana Zunge), riechende (ghraāa $\sim$ Nase) - sind Strömungen der Menschen als Mündungen nach außen.' Diese körperlichen Beobachtungen der Wundärzte entsprechen den durchlaufenden Einschätzungen der Sinne als Äußerungen oder Ausstrahlungen.

(10) ,Die fünf Sinnesziele sind Ton (śabda), Getast (sparśa), Ansicht (rüpa), Geschmacks-Saft (rasa), Geruch (gandha). Die Bezeichnung artha = Ziel verleitet zu einem modern durchaus berechtigten «objektiven» Sammelbegriff [wie schon zuvor dargelegt], welcher aber nicht der indischen Denkweise entspricht. So ist auch rüpa, üblich als «Aussehen, Form, Gestalt, Farbe» erklärt, besser

16 Wörterheft Nr. 105, in Mitt. Inst. Orientforsch. 8 (Berlin 1961) S. 105, ausführlicher im P H M A - Heft demnächst.

17 Osiris 12 (Brugis 1956) 467.

18 Nova Acta Leopoldina, N.F. Nr. 115, zumal S. 22 (Leipzig 1955). 
durch ,Ansicht ${ }^{6}$ zu übertragen, während bei den ,Zielen ${ }^{6}$ rasa den zugrunde liegenden Begriff ,Saft ${ }^{6}$ einschließt.

(11) Die fünf Sinnesbesinnungen sind Blickbesinnung usw. [d.h. durch Behorchen, Beriechen, Schmecken, Betasten, wie in 7]. Da buddhi als «Einsicht, Verstand, Geist» oder dergleichen angesprochen wird, so kann seine Einreihung unter die Sinne überraschen. Die Herleitung des Ausdruckes von budh- $=\ll$ erwachsen, zur Besinnung kommen» begünstigt die Übertragung durch ,Besinnung6.

Am merkwürdigsten mutet an, daß das Denken zu den Sinnen zugerechnet wird, zumal in der Einleitung 4: ,Der Obersinn ist nun das Denken (manas), welcher auch sattva heißt, einige nennen ihn auch cetas.' Cetas (üblich: «Einsicht, Bewußtsein» oder dergleichen) und sattva (Wesenheit) haben nach dem Lehrtext wegen der Gleichung doch als Denken zu gelten. Die Zustimmung im Kommentar ist etwas bedingt:, Wenn man der Meinung eines anderen nicht entgegentritt, so stimmt man zu.' Aber bei den sogenannten Geisteskrankheiten besteht ein Austausch dieser drei Bezeichnungen ${ }^{19}$, so daß eine einheitliche Bedeutung darnach begründet erscheint.

Das Denken aber, was in 4 als ,Obersinn' betont ist, und auch die Besinnung wird dann in 12 in eine Überschicht gezogen zum Selbst (ãtman) und U̇berselbst (adhyätma), wie zu einem Seelenbegriff. Dieser wurde in den ärztlichen Sammelwerken mannigfach ausgebildet ${ }^{20}$, ist aber zur vorliegenden Aufgabe hier nicht darzulegen. In der Caraka-Samhitā śärīra ${ }^{\circ} 1$ wird zwar Denken und Besinnung auch jener Überschicht zugewiesen, jedoch beide in den Sinnesschilderungen ab 18 noch mit diesen vereinigt, wobei (wie in sütra ${ }^{\circ} 8,7-11$ ) Unordnungen in den gegenseitigen Beziehungen und Bezeichnungen bestehenblieben. In SuśrutaSamhitā śărīra ${ }^{\circ}$ 1, 4 ist das Denken sogar den Sinnesgruppen nur angehängt und in der Überschicht gar nicht erwähnt. Diese Regelwidrigkeiten deuten an, daß entsprechende Ausrichtungen in Auffassungen der indischen Ärzte im Hintergrund blieben. Wenn in Caraka-Samhitā śärïra ${ }^{\circ} 1,32$ gesagt wird: ,Wie der Ton gesehen wird ${ }^{6}$, so ist damit das gültige und beherrschende Muster vom Sehen nachweisbar, das auch einmal bildlich als Ausstrahlung ${ }^{21}$ überliefert ist. Gewiß ist in unmittelbaren Schilderungen der sinnlichen Erfahrungen diese Ausrichtung nicht immer erkennbar, die als eine durchlaufende indisch-wissenschaftliche Grundlage zu beurteilen ist. Auch das «Aufleuchten des Augenstrahls »22 in der

19 Indo-Iranian Journal 3 ('s-Gravenhage 1959) 277, wo auch medizingeschichtlich zur Bedeutung von cetas zuvor Stellung genommen wird.

20 Nova Acta Leopoldina, N.F. Nr. 138 (Leipzig 1958) 102-127.

21 A.v.LeCoq und E. Waldschmidt, Die buddhistische Spätantike in Mittelasien, T.7, S. 49f., Tafel 17 (Berlin 1933).

22 Trübners Deutsches Wörterbuch, Band 1, S. 360 b (Berlin 1939). 
modernen Ausdrucksweise kann hierbei nicht untersucht werden auf eine Frage, ob ein Zusammenhang oder ein Gemeinplatz annehmbar ist. Doch die Zusammenfassung in Caraka-Samhitā sūtra ${ }^{\circ}$, 16, welche der Kommentar aus diesem Lehrtext zu dem sogenannten Seelenbegriff in 12 vorzieht, erscheint als wichtig: manasastu cintyamarthah $=$ Des Denkens Ziel ist das zu Bestrahlende 6 . Sprachwissenschaftlich wird zwar cintya als, zu denken" aufgefaßt; damit würde aber das Denken ausdrücklich nur wiederholt, aber nicht erklärt. Medizingeschichtlich ist daher zu folgern, daß auch hier die beherrschende Wertung des Sehens als eine ausstrahlende Äußerung der Sinnesvermögen bei ihrer Übernahme zu seelenartigen Vorstellungen zugrunde liegt. 